Revista Mexicana de Astronomía y Astrofísica, Volumen 3, Mayo 1977

\title{
ETOILES MULTIPLES ET AMAS OUVERTS
}

\author{
J. Dommanget \\ Observatoire Royal de Belgique
}

\begin{abstract}
RESUMEN
Generalmente se tiene la sospecha que estrellas múltiples y cúmulos galácticos son objetos de diferente clase. Sus distribuciones de frecuencia como función del número de componentes, $n$, así como su tamaño y masa, muestran diferencias importantes entre las dos categorías, y también la existencia probable de un límite que queda entre $\mathrm{n}=10 \mathrm{y}$ $\mathrm{n}=20$ componentes. La diferencia básica entre estos objetos aparentemente es, que los sistemas múltiples parecen ser finalmente componentes del medio estelar de la misma manera que estrellas solas y dobles, mientras que los cúmulos galácticos son grupos de componentes de una región particularmente densa de este medio.
\end{abstract}

\section{ABSTRACT}

It is generally suspected that multiple systems and galactic clusters are objects of different kinds. Their frequency distribution as a function of the number of components, $n$, as well as their sizes and their masses, show important differences between both categories and also the probable existence of a limit lying between $n=10$ and $n=20$ components. The basic difference between these objects seems to be that the multiple systems finally appear to be components of the stellar medium in th same way as single and double stars, whereas the galactic clusters are groups of components of a particularly dense part of this medium.

\section{INTRODUGTION}

La définition de certains objets célestes pose parfois un problème plus ardu qu'il ne paraît à priori. Il en va ainsi des couples stellaires au sujet desquels, dès 1911, R. G. Aitken réunissait 22 experts décidés à se pencher sur la recherche d'une définition précise de ces objets (Aitken 1911).

En 1957, lors du Colloque U.A.I. "On the Evolution of Double Stars" tenu à Bruxelles, nous avons proposé pour définition de l'étoile double: "association physique permanente de deux étoiles" en entendant pour la qualité de "permanente", una échelle de temps supérieure d'un ordre de grandeur au moins, à la période de révolution (Dommanget 1967).

Ceci étant, on ne recontre aucune difficulté ensuite -en principe du moins-- à distinguer les couples répondant à cette condition, c'est-à-dire les étoiles binaires, des couples optiques ou des couples d'étoiles passagèrement associées par une recontre fortuite dans l'espace. L'étoile binaire présente, suivant cette définition, une orbite nécessairement elliptique et une énergie totale évidemment négative.

Dans le cas des étoiles multiples, il se pose un problème de définition analogue. Pour les systèmes triples, le problème n'est sans doute pas plus difficile à traiter que pour les systèmes binaires ainsi que d'ailleurs, pour les systèmes multiples ayant un nombre de composantes un peu plus grand. En effet, en généralisant la définition proposée pour les binaires, il semble qu'une définition adéquate des systèmes multiples devrait revenir à imposer pour l'énergie totale, une valeur toujours négative par exemple.

Toutefois, si le nombre de composantes devient très grand, on ne parle plus de systèmes ou étoiles multiples, mais bien d'amas, ouverts ou globulaires, et d'associations.

Mais ce changement de terminologie, reflète-t-il 
une différence profonde entre ces divers objets ou bien, les amas et les associations constituent-ils -dans une certaine mesure du moins- des prolongements du domaine des étoiles multiples vers les objets à grands nombres de composantes?

Pour diverses raisons évidentes quant aux caractéristiques de ces divers objets (spectres, origines probables, etc.) seuls les amas ouverts offrent, à priori, la possibilité de constituer un tel prolongement.

Il se pose donc le problème de définir les systèmes multiples en même temps que les amas ouverts.

Comme on n'a pu étudier suffisamment de systèmes multiples et d'amas ouverts dans le détail, du point de vue dynamique, et connaître de ce fait, les valeurs des énergies totales correspondantes, il reste à envisager d'autres voies d'exploration que nous nous proposons de décrire. Celles-ci portent sur la fréquence, la dimension et la masse des systèmes d'étoiles.

\section{LA FREQUENCE DES SYSTEMES STELLAIRES}

\section{a) Systèmes Multiples}

On sait que la fréquence des systèmes multiples décroît très rapidement avec l'accroissement du nombre $\mathrm{n}$, des composantes. Ce fait est illustré par les nombres figurant dans le Tableau 1 et donnant les fréquences relatives de ces divers systèmes que l'on recontre dans l'Index Catalogue (Jeffers and van den Bos 1963). Ces fréquences doivent cependant être corrigées tout d'abord parce qu'elles n'ont été établies qu'à partir des duplicités et mutiplicités

TABLEAU 1

FREQUENCES DES.SYSTEMES MULTIPLES

\begin{tabular}{cccccc}
\hline & $\begin{array}{c}\text { Index } \\
\text { Cat. }\end{array}$ & $\begin{array}{c}\text { A. H. } \\
\text { BATTEN }\end{array}$ & $\begin{array}{c}\text { C. E. } \\
\text { WORLEY }\end{array}$ & $\begin{array}{c}\text { P. } \\
\text { BAIZE }\end{array}$ \\
\hline 2 & $90,8 \quad \%$ & 66,7 & $\%$ & $78,1 \%$ & $86,9 \%$ \\
3 & 7,2 & 25,0 & 17,4 & 11,2 \\
4 & 1,4 & 6,7 & 3,4 & 1,4 \\
5 & 0,34 & 1,4 & 0,9 & 0,23 \\
6 & 0,11 & 0,2 & 0,4 & 0,23 \\
7 & 0,05 & 0,03 & - & - \\
8 & 0,017 & 0,004 & - & - \\
9 & 0,009 & 0,001 & - & - \\
\hline
\end{tabular}

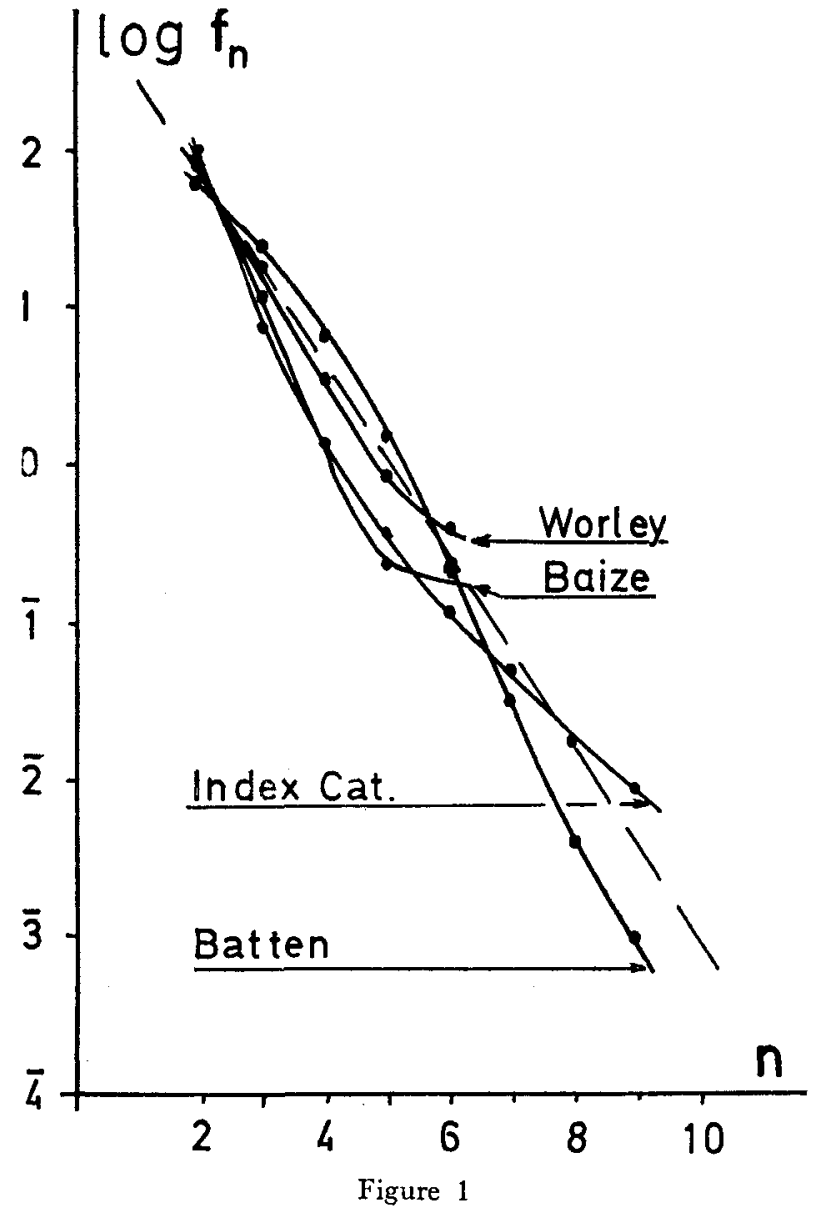

visuelles, sans tenir compte des duplicités spectroscopiques et photométriques éventuelles des composantes et, par ailleurs, parce qu'un grand nombre de composantes de systèmes multiples sont optiques.

D'après une étude de $\mathrm{A}$. $\mathrm{H}$. Batten, basée sur le sixième catalogue d'orbites d'étoiles doubles spectroscopiques, la fréquence des systèmes ayant au moins $n$ composantes, pourrait être finalement proportionnelle à $1 / n$ ! (Batten 1967). D'après cette expression, on trouve pour fréquences des systèmes multiples, les valeurs données dans le Tableau 1.

Ces valeurs paraissent convenablement confirmées d'une part par une recherche statistique effectuée par Worley (1967 à partir de son catalogue d'orbites d'étoiles doubles visuelles, ainsi que d'autre part, par un relevé statistique par Baize (1975) parmi toutes les étoiles situées à moins de 25 parsecs du Soleil et qui dès lors constituent un échantillon particulièrement bien exploré. 
TABLEAU 2

\begin{tabular}{crrrrrrrrrrrrrr}
\hline $\mathrm{n}$ & $\mathrm{de}$ & 0 & 10 & 20 & 30 & 40 & 50 & 60 & 70 & 80 & 90 & 100 & 110 & 120 \\
& $\mathrm{a}$ & 10 & 20 & 30 & 40 & 50 & 60 & 70 & 80 & 90 & 100 & 110 & 120 & 130 \\
\hline $\mathrm{N}$ & 1 & 23 & 52 & 36 & 23 & 39 & 17 & 6 & 9 & 0 & 10 & 0 & 7 \\
$\mathrm{f}(\%)$ & 0,4 & 10,3 & 23,3 & 16,1 & 10,3 & 17,5 & 7,6 & 2,7 & 4,0 & 0,0 & 4,5 & 0,0 & 3,1 \\
\hline
\end{tabular}

Finalement, il semble, d'aprês la Figure 1, sur laquelle ont été portées les données du Tableau 1, que l'on puisse représenter assez bien les logarithmes des fréquences relatives probables $f_{n}$ (en $\%$ ), des systèmes multiples $(2 \leqslant n \leqslant 9)$, par la droite tracée en trait interrompu.

\section{b) Amas Ouverts}

En ce qui concerne les amas ouverts, nous n'avons trouvé qu'une seule liste -ancienne malheureusement- de 223 amas, donnant le nombre d'étoiles estimé pour chacun d'eux (Shapley 1933). A partir de cette liste, nous avons établi le Tableau 2.

La figure 2 représente les logarithmes des pourcentages en fonction de $\mathrm{n}$ ainsi que la droite trouvée en a) pour les étoiles multiples.

On constate que les courbes relatives aux étoiles multiples d'une part et aux amas ouverts de l'autre, présentent des allures totalement différentes particulièrement en ce qui concerne leurs pentes. Toutefois, elles ne sont pas directement comparables l'une à l'autre car elles ont été établies séparément et sur la base de relevés statistiques portant sur des volumes d'espace différents (celui de l'Index Catalogue d'une part et celui d'une liste des amas ouverts connus à une certaine époque de l'autre) et aussi, parce qu'elle porte sur des fréquences relatives à des intervalles

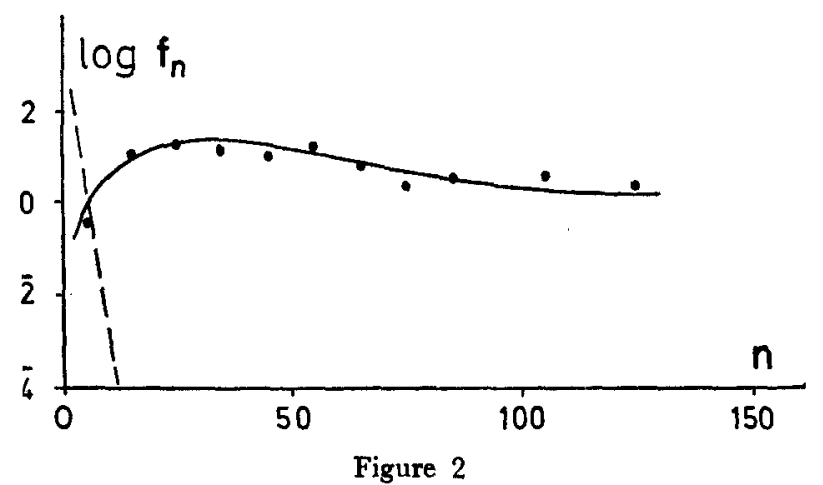

differents en $n$. Aussi, dans une statistique générale de tous ces objets célestes observables dans un même volume d'espace, la partie de la courbe de fréquence correspondant aux grandes valeurs de $n$, se situerait certainement plusieurs unités plus bas en $\log f_{n}$, dans la Figure 2, que celle trouvée ici pour les amas ouverts.

Mais les deux courbes de cette figure permettent d'imaginer la forme générale d'une telle courbe de fréquence globale et de prévoir l'existence d'une différence d'allure très notable entre sa partie correspondant aux systèmes ayant de 2 à 10 composantes et celle correspondant aux systèmes en comportant au moins une vingtaine. On observerait donc dans la zone allant de $n^{\prime}=10$ à $n=20$ environ, soit une discontinuité assez marquée, soit au moins, une variation très rapide de la pente de la courbe de fréquence, indice d'une limite susceptible de séparer là, les étoiles multiples des amas ouverts.

\section{LES DIMENSIONS DES SYSTEMES STELLAIRES}

\section{a) Systèmes Multiples}

Par notre définition du couple stellaire basée sur la "permanence" de la liaison physique de leurs composantes, nous avons trouvé que les dimensions les plus grandes de ces objets devaient avoisiner:

$$
\mathrm{D}=0,01 \text { parsec }
$$

Pour les systèmes triples, quadruples, etc, il est plus difficile de conclure aussi immédiatement à une limite similaire. On conçoit cependent que plus le nombre d'étoiles composant le système est grand, plus sa pérennité est assurée en tant que système multiple, vis-à-vis des perturbations susceptibles d'être produites par le rapprochement passager d'une étoile 


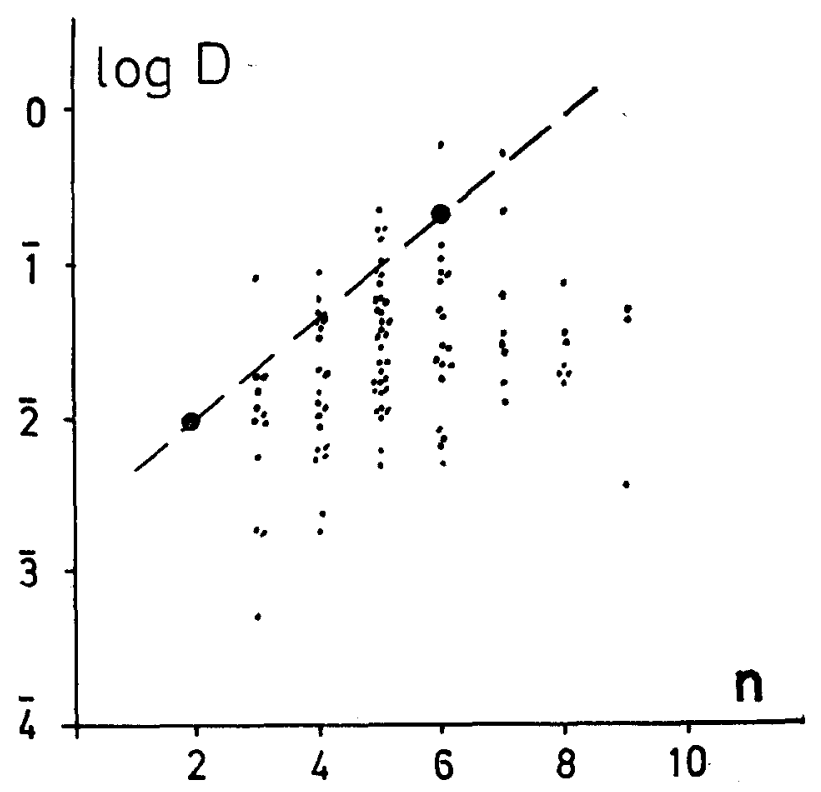

Figure 3

environnante. Aussi, plus le nombre des composantes est grand, plus les dimensions limites des systèmes "permanents" peuvent être importantes.

Le graphique de la Figure 3 porte les dimensions:

i) d'un échantillon des systèmes triples, quadruples et quintuples, figurant dans l'Index Catalogue (Jeffers and van den Bos 1963),

ii) de tous les systèmes multiples comportant de 6 à 9 composantes figurant dans le même catalogue, et pour lesquels, une parallaxe est connue.

Ces systèmes comportent sans doute des composantes optiques que nous n'avons pas pu détecter et éliminer et, par ailleurs, des couples serrés (spectroscopiques) inconnus. Aussi, la droite tracée sur cette figure n'est qu'une approximation de la limite supérieure réelle en $\log \mathrm{D}$ à laquelle on peut s'attendre.

Toutefois, on remarquera que la limite: $\mathrm{D}=0,01$ parsec que nous avons proposée pour les systèmes binaires "permanents" et celle de 0,2 parsec, considérée arbitrairement par Allen et Poveda (1974) pour les systèmes sextuples du type "trapèze", sont en accord parfait avec cette limite que nous adopterons comme approximation suffisante pour la suite des discussions.

\section{b) Amas Ouverts}

Un graphique similaire (Figure 4) a été établi pour les amas ouverts à partir d'une liste de 90 amas dressée à l'aide des tableaux figurant dans les travaux de Shapley (1933) et de Schmidt (1963). Les

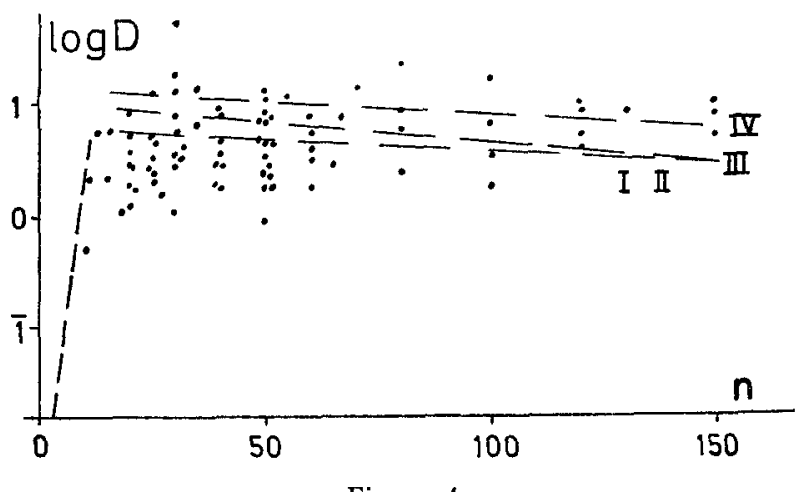

Figure 4

valeurs moyennes données dans l'ouvrage de LandoltBörnstein (1965) pour les classes de concentration I, II, III et IV de Trumpler, sont également représentées dans ce graphique où nous avons porté la droite limite déterminée à la Figure 3.

On remarque ici également, que les domaines occupés par les étoiles multiples d'une part et par les amas ouverts de l'autre, se "raccordent" par une variation très rapide dans la courbe exprimant les dimensions extrèmes des objets, en fonction de $\mathrm{n}$, dans la région allant de $n=10$ à $n=20$ environ.

\section{LES MASSES DES SYSTEMES STELLAIRES}

La Figure 5 donne, en fonction de $\mathbf{n}$, les masses totales des 90 amas ouverts de la liste citée ci-dessus.

Alors que les masses sont assez bien définies, les nombres $\mathrm{n}$ le sont beaucoup moins bien, mais les erreurs qui les entachent -même très importantesn'affectent pratiquement pas la distribution observée des points dans le diagramme: les masses des amas restent comprises à peu près entre 200 et 10000 masses solaires et cela quasi indépendamment de $\mathrm{n}$.

Dans le cas des étoiles multiples (n petit), les composantes ont, en moyenne, des masses du même ordre que celles observées dans les systèmes doubles 


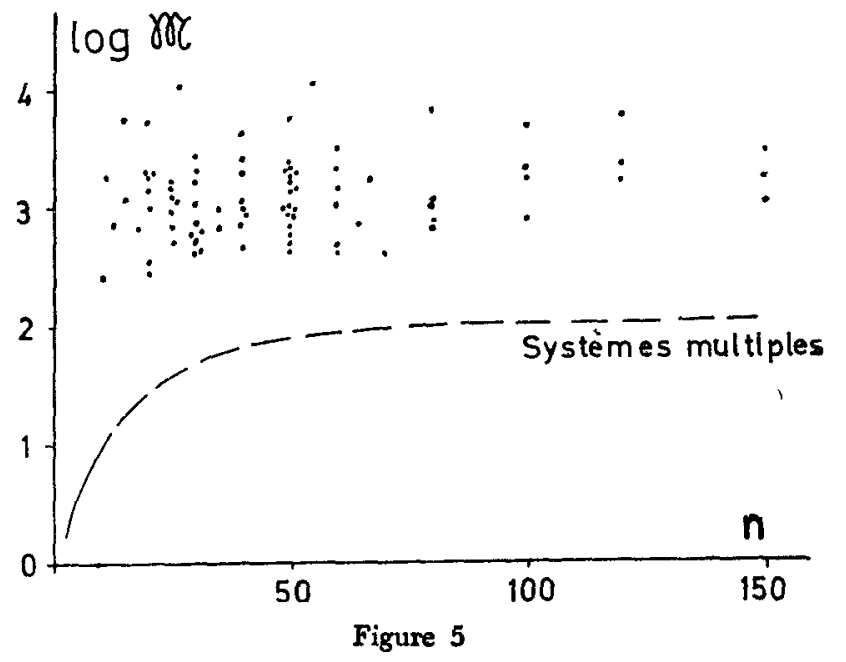

et valant en moyenne: $1,2 \mathrm{M}_{\odot}$. Les masses totales des étoiles multiples peuvent donc être estimés, également en moyenne, à:

$$
\mathrm{n} \times\left(1,2 \mathrm{M}_{\odot}\right)
$$

La courbe représentant le logarithme de la masse totale ainsi calculée, en fonction de $\mathrm{n}$ a été tracée en trait plein sur la Figure 5 et l'on voit que son prolongement (en trait interrompu) vers les valeurs de $n$ plus grandes que 10 environ, ne représente nullement les masses connues pour les amas ouverts. Un certain accord entre cette courbe et les points représentatifs de ces amas, ne pourrait être obtenue qu'en choisissant une masse stellaire moyenne de l'ordre de 10 à $100 \mathrm{M}_{\odot}$.

De ce point de vue, une distinction supplémentaire doit être faire entre les systèmes multiples comportant au maximum une dizaine de composantes et les amas ouverts formés d'un beaucoup plus grand nombre d'etoiles.

\section{v. CONGLUSION}

Ces quelques considérations que nous venons de développer, confirment l'opinion de certains auteurs, en particulier de Batten (1967), comme quoi, les systèmes multiples et les amas ouverts sont des objets célestes de natures différentes et dès lors probablement aussi d'origines différentes.

Il semblerait tout d'abord que les systèmes multiples soient des ensembles d'une dizaine de composantes au plus; les amas, des groupements de plusieurs dizaines à plusieurs centaines d'étoiles. De plus, les dimensions des étoiles multiples étant d'un ordre de grandeur inférieur à celui des distances moyennes séparant les étoiles du milieu stellaire normal, alors que celles des amas les dépassent nettement, les premiers devraient être considérés -comme les étoiles simples et les étoiles doubles - parmi les objets constituant le milieu stellaire tandis que les amas constitueraient plutôt des portions de ce milieu, portions à densités souvent particulièrement élevées.

Ainsi la distinction terminologique entre systèmes multiples et amas prendrait une signification encore plus profonde.

Il serait toutefois souhaitable que soit établi sur la base des données d'observation les plus récentes, une liste détaillée des amas donnant en particulier, les nombres d'étoiles qu'ils contiennent, leurs dimensions et leurs masses, afin de mieux assurer les conclusions ci-dessus.

Une étude détaillée des systèmes de 10 à 20 étoiles serait également du plus haut intérêt.

\section{BIBLIOGRAPHIE}

Aitken, R. G. 1911, Astr. Nach., 188, 281.

Allen, C., and Poveda, A. 1974, Proc. IAU Symposium No. 62, The Stability of the Solar System and of Small Stellar Systems, ed. Y. Kozai (Dordrecht: D. Reidel), 239.

Baize, P. 1975, L'Astronomie, 89, 159.

Batten, A. H. 1967, Comm. Obs. R. Belgique, 17, 218.

Dommanget, J. 1967, Comm. Obs. R. Belgique, 17, 25.

Jeffers, H. M., and van den Bos, W. H. 1963, Index Catalogue of Visual Double Stars, Pub. Lick Obs., 21. Landolt-Börnstein 1965, 1, 594.

Schmidt, K. H. 1963, Astr. Nach., 287, 41.

Shapley, H. 1933, Handbuch der Astrophysik, 5, 698.

Worley, C. E. 1967, Comm. Obs. R. Belgique, 17, 221. 


\section{DISCUSSION}

Abt: In your last diagram showing mases of open clusters and visual multiples, the open clusters, contain mostly B and A stars, while the visual multiples are assumed to have 1-2 $\mathbf{M}_{\odot}$. Therefore the separation in mass is by definition and not a real difference. You should compare B-type visual multiples with open clusters.

Aarseth: Recent works by Oort and collaborators gives a dynamical mass of about $250 \mathrm{M}_{\odot}$ for the Hyades, with some 400 known members. Hence the corresponding mean mass would not exceed about $0.6 \mathrm{M}_{\odot}$.

Scarfe: How does observational selection affect the number of clusters with relatively few members? Such clusters might be hard to detect.

Dommanget: Clusters with relatively few members must be of a small size and should appear as multiple systems and not as open clusters. They should thus have been taken into account in the statistics of multiple star systems. Therefore observational selection may not affect that number more than the number of the corresponding, multiple system.

King: I agree with Abt's comment that the clusters considered are different from the binaries, because their mass is largely in B stars. There is an observational selection in favor of $\mathrm{OB}$ clusters. I also agree with Scarfe that there is a selection in $n$ for clusters. Furthermore, the values of $\mathbf{n}$ given in these catalogs are almost totally meaningless for your purpose, since they refer only to an arbitrary number of brighter stars, which is only a small fraction of the total number of stars in the cluster. I would also comment that the masses to which you referred are not dynamical but calculated rather from a mass-luminosity relation. Dynamical mass determinations exist for very few clusters.

Finally, I should like to ask where your frequency of eight-star systems came from. I thought that people this morning agreed that the richest multiples had six stars, or pos. sibly seven. 